University of Texas Rio Grande Valley

ScholarWorks @ UTRGV

Physics and Astronomy Faculty Publications and Presentations

$10-10-2016$

\title{
AN ECCENTRIC BINARY MILLISECOND PULSAR with A HELIUM WHITE DWARF COMPANION in the GALACTIC FIELD
}

John Antoniadis

David L. Kaplan

Kevin Stovall

Paulo C.C. Freire

Julia S. Deneva

See next page for additional authors

Follow this and additional works at: https://scholarworks.utrgv.edu/pa_fac

Part of the Astrophysics and Astronomy Commons

\section{Recommended Citation}

John Antoniadis, et. al., (2016) AN ECCENTRIC BINARY MILLISECOND PULSAR with A HELIUM WHITE DWARF COMPANION in the GALACTIC FIELD.Astrophysical Journal830:1. DOI: http://doi.org/10.3847/ 0004-637X/830/1/36

This Article is brought to you for free and open access by the College of Sciences at ScholarWorks @ UTRGV. It has been accepted for inclusion in Physics and Astronomy Faculty Publications and Presentations by an authorized administrator of ScholarWorks @ UTRGV. For more information, please contact justin.white@utrgv.edu, william.flores01@utrgv.edu. 


\section{Authors}

John Antoniadis, David L. Kaplan, Kevin Stovall, Paulo C.C. Freire, Julia S. Deneva, Detlev Koester, Fredrick Jenet, and Jose G. Martinez

This article is available at ScholarWorks @ UTRGV: https://scholarworks.utrgv.edu/pa_fac/88 


\title{
AN ECCENTRIC BINARY MILLISECOND PULSAR WITH A HELIUM WHITE DWARF COMPANION
} IN THE GALACTIC FIELD

\author{
John Antoniadis $^{1}$, David L. Kaplan ${ }^{2}$, Kevin Stovall $^{3}$, Paulo C. C. Freire ${ }^{4}$, Julia S. Deneva ${ }^{5}$, \\ Detlev Koester ${ }^{6}$, Fredrick Jenet ${ }^{7}$, and Jose G. Martinez ${ }^{4}$ \\ ${ }^{1}$ Dunlap Institute for Astronomy \& Astrophysics, University of Toronto, 50 St. George Street Toronto, Ontario M5S 3H4, Canada; antoniadis@dunlap.utoronto.ca \\ ${ }^{2}$ Department of Physics, University of Wisconsin-Milwaukee, 1900 East Kenwood Boulevard, Milwaukee, WI 53211, USA \\ ${ }^{3}$ Department of Physics and Astronomy, University of New Mexico, Albuquerque, NM 87131, USA \\ ${ }^{4}$ Max-Planck-Institut für Radioastronomie, Auf dem Hügel 69, D-53121 Bonn, Germany \\ ${ }^{5}$ National Research Council, resident at the Naval Research Laboratory, 4555 Overlook Ave SW, Washington, DC 20375, USA \\ ${ }^{6}$ Institut für Theoretische Physik und Astrophysik, Christian-Albrechts-Univeristät Kiel, D-24098 Kiel, Germany \\ ${ }^{7}$ Center for Advanced Radio Astronomy, Department of Physics and Astronomy, University of Texas at Brownsville, Brownsville, TX 78520, USA \\ Received 2016 January 28; revised 2016 June 13; accepted 2016 June 17; published 2016 October 7
}

\begin{abstract}
Low-mass white dwarfs (LMWDs) are believed to be exclusive products of binary evolution, as the universe is not old enough to produce them from single stars. Because of the strong tidal forces operating during the binary interaction phase, the remnant systems observed today are expected to have negligible eccentricities. Here, we report on the first unambiguous identification of an LMWD in an eccentric $(e=0.13)$ orbit around the millisecond pulsar PSR J2234+0511, which directly contradicts this picture. We use our spectra and radio-timing solution (derived elsewhere) to infer the WD temperature $\left(T_{\text {eff }}=8600 \pm 190 \mathrm{~K}\right)$, and peculiar systemic velocity relative to the local standard of rest $\left(\simeq 31 \mathrm{~km} \mathrm{~s}^{-1}\right)$. We also place model-independent constraints on the WD radius $\left(R_{\mathrm{WD}}=0.024_{-0.002}^{+0.004} R_{\odot}\right)$ and surface gravity $\left(\log g=7.11_{-0.16}^{+0.08} \mathrm{dex}\right)$. The WD and kinematic properties are consistent with the expectations for low-mass X-ray binary evolution and disfavor a dynamic three-body formation channel. In the case of the high eccentricity being the result of a spontaneous phase transition, we infer a mass of $\sim 1.60 M_{\odot}$ for the pulsar progenitor, which is too low for the quark-nova mechanism proposed by Jiang et al., and too high for the scenario of Freire \& Tauris, in which a WD collapses into a neutron star via a rotationally delayed accretion-induced collapse. We find that eccentricity pumping via interaction with a circumbinary disk is consistent with our inferred parameters. Finally, we report tentative evidence for pulsations that, if confirmed, would transform the star into an unprecedented laboratory for WD physics.
\end{abstract}

Key words: Galaxy: stellar content - pulsars: individual (PSR J2234+0611) - pulsars: general - stars: neutron white dwarfs

\section{INTRODUCTION}

Millisecond radio pulsars (MSPs) are a distinct population of neutron stars (NSs) with fast spin periods and magnetic fields several orders of magnitude weaker than those of "normal" pulsars. These properties are thought to reflect a long mass transfer episode from a stellar companion. In the standard "recycling" scenario, the donor's mass accumulated on the NS increases its spin frequency and, through a process not yet well understood, buries the magnetic field (see Taam \& van den Heuvel 1986; Bhattacharya \& van den Heuvel 1991). Depending on the orbital period and initial donor mass, the remnant companions are usually low-mass white dwarfs (LMWDs; with $\left.0.16-0.4 M_{\odot}\right)$, or semi-degenerate stars with masses in the range 0.001-0.4M $\odot$ (e.g., Archibald et al. 2009; Tauris 2011). As a result of efficient tidal dissipation during the mass-transfer episode, the post-interaction orbits are expected to be extremely circular $\left(e \sim 10^{-7}-10^{-3}\right)$, which is indeed the case for most MSPs in the Galactic field (Phinney 1992).

Surprisingly, recent surveys with the Arecibo, Effelsberg, and Parkes radio telescopes led to the discovery of five peculiar binary MSPs with mass functions indicative of low-mass companions, but large eccentricities of $e \simeq 0.025-0.13$ (henceforth eMSPs). The only previously known eccentric MSP in the Galactic field, PSR J1903+0327 (Champion et al. 2008; Freire et al. 2011) has a substantially higher eccentricity $(e \simeq 0.44)$ and a $1 M_{\odot}$ main-sequence companion. As the latter could not have been responsible for recycling the pulsar, the binary is most likely the remnant of a triple, where the least massive star, responsible for recycling the MSP, was ejected as a result of unstable orbital evolution (Bejger et al. 2011; Freire et al. 2011; Portegies Zwart et al. 2011; Perets \& Kratter 2012; Pijloo et al. 2012).

The recently identified eMSPs are qualitatively different from PSR J1903+0327. The binary companions have very low masses, suggesting that they most likely descend from the donors that spun-up the pulsars. In addition, their orbits resemble each other in many ways, making an origin in a triple system unlikely because the chaotic disruption of the original systems would also result in a broad range of orbital properties for the observed remnant binaries.

An attractive alternative scenario is that eMSPs are the direct descendants of low-mass X-ray binaries (LMXBs). This hypothesis places strong constraints both on the expected observational properties and on the formation mechanism. For LMXBs with sufficiently large initial separations, the recycling episode starts when the companion evolves off the main sequence. In red giants, the mass of the helium core is proportional to the stellar radius, which in turn is regulated by the orbital separation. Therefore, the final companions are expected to be LMWDs with masses proportional to the orbital period (Tauris \& Savonije 1999). In addition, because tidal dissipation acts on sub-thermal timescales, the eccentricities 


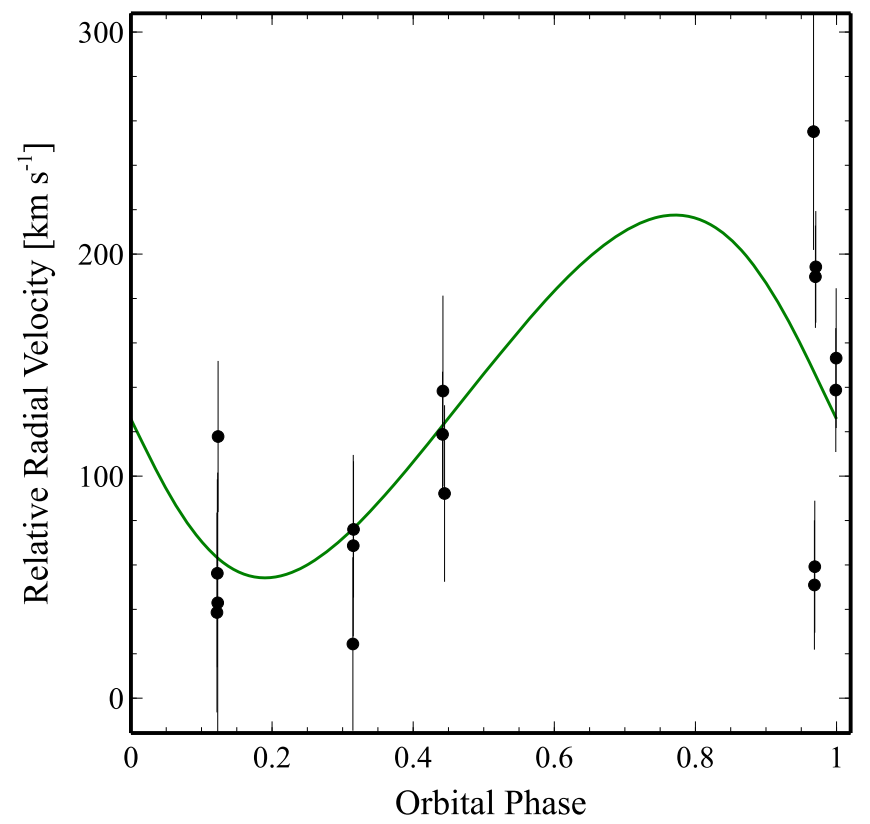

Figure 1. Radial velocities of the companion to PSR J2234+0611 as a function of the orbital phase. The solid line shows the inferred best-fit orbit.

must have increased either after or during the very last phase of the long-term recycling episode.

A possible explanation for the observed eccentricities is a spontaneous phase transition of either a super-Chandrasekhar WD collapsing into an NS (Freire \& Tauris 2014), or of an NS imploding into a strange-quark star (Jiang et al. 2015). In either case, the transformation is mass-critical and therefore the pulsar masses should be similar. An alternative mechanism could be the interaction of the post-LMXB system with a circumbinary disk (Antoniadis 2014), in which case the pulsar masses need not be similar. A comprehensive comparison of the aforementioned scenarios and their predictions is given in Table 1 and discussed further in Section 4.

In this work we present optical observations of PSR J2234 +0611 (Deneva et al. 2013), a nearby eMSP with a 32 day orbital period and an eccentricity of 0.13. Our spectroscopy unambiguously confirms that the companion is an LMWD, making PSR J2234+0611 the first such system identified in the Galactic field. The paper is structured as follows: In Section 2 we describe our data set and analysis. In Section 3 we present constraints on the WD and NS mass and finally, in Section 4 we explore the ramifications for the proposed formation theories. We conclude in Section 5.

\section{OBSERVATIONS}

\subsection{Radio Timing}

The radio timing of the pulsar is presented in a companion paper by K. Stovall et al. (in preparation; Paper II). Here, we summarize some of the key results for completeness.

The timing baseline, which now spans over two years, allows for a precise determination of the proper motion, $\left[\mu_{\alpha}, \mu_{\delta}\right]=[25.390(27), 9.48(0.06)] \mathrm{mas} \mathrm{yr}^{-1}$, and the parallax, $\varpi=0.742$ (28) mas, placing the system at a distance of $1.35(5) \mathrm{kpc}$. In addition, the combined constraints on relativistic periastron precession and Shapiro delay, yield a mass of $M_{\mathrm{c}}=0.275(8) M_{\odot}$ for the companion and $M_{\mathrm{PSR}}=1.39(1) M_{\odot}$ for the pulsar. In Sections 3 and 4, we use these measurements to constrain the size of the companion, compute the orbit of the system in the Galaxy, and explore the implications for the proposed formation scenarios.

\subsection{Optical Observations}

The optical counterpart to PSR J2234+0611 was first identified in the SDSS archive (Eisenstein et al. 2011) as a faint star $(g=22.17)$ coincident with timing position of the pulsar. Given the small stellar density of the field, the probability for a chance alignment is low $(<0.001 \%)$. Based on the measured SDSS ugriz magnitudes, the parallax distance, and a collection of WD cooling models (e.g., Serenelli et al. 2001; Antoniadis et al. 2013), we find that the star is consistent with being an LMWD with a temperature of $T_{\text {eff }} \simeq 8500 \mathrm{~K}$.

We followed up PSR J2234+0611 spectroscopically using the FORS2 instrument (Appenzeller et al. 1998) of the Very Large Telescope in Chile. All observations were carried out in service mode between 2014 July and September using the MIT red-sensitive camera, which delivers a resolution of 0.25 arcsec per binned-by-two pixel along the spatial direction. We used the 1200 lines $\mathrm{mm}^{-1}$ grism (GRIS_1200B+97), which covers the spectral range between 378 and $510 \mathrm{~nm}$ with a resolution of $0.072 \mathrm{~nm} \mathrm{pix}^{-1}$. A total of $26,1420 \mathrm{~s}$ spectra were collected through a 1 arcsec slit, which was rotated by 31.3 (north to east) in respect to the parallactic angle to include a bright reference star south-west of the target. In addition, we recorded two $1300 \mathrm{~s}$ exposures using a 5 arcsec slit for calibration purposes. The atmospheric conditions were generally variable, but some exposures were taken during clear conditions. The seeing ranged from 0.6 to 1.5 arcsec.

The data were reduced using astropy (Astropy Collaboration et al. 2013) ${ }^{8}$ and custom python routines. First, we removed the bias level using the median estimate from the overscan region. Then, we corrected for small-scale sensitivity variations using lamp exposures, normalized both along the spatial and the dispersion directions. The sky level was estimated by using a second-degree polynomial fitted to 100 arcsec regions on either side of the target and the reference, but excluding 5 arcsec areas around them. Finally, we extracted the spectra using an optimal-weighting method similar to that of Horne (1986).

The dispersion solution was derived using a second-degree polynomial fit to the $\mathrm{CuAr}$ lamp spectra, recorded at the end of each run. The solutions have root-mean-square (rms) residuals ranging from $\sim 0.02$ to $0.04 \mathrm{~nm}$ for 18 to 20 lines. For flux calibration, we first corrected for wavelengthdependent slit losses using the normalized wide-to-narrow slit flux ratio of the reference star and then applied the instrument response derived using an exposure of a standard star on a photometric night.

\section{RESULTS}

\subsection{Radial Velocities and Constraints on the Mass Ratio}

Radial velocities were measured using the method described in Antoniadis et al. (2012). First, we identified the reference star as being a K3V star and used a medium-resolution PHOENIX spectrum as a template to measure its velocity (Husser et al. 2013). For PSR J2234+0611, we first fitted a grid

\footnotetext{
8 http://www.astropy.org
} 


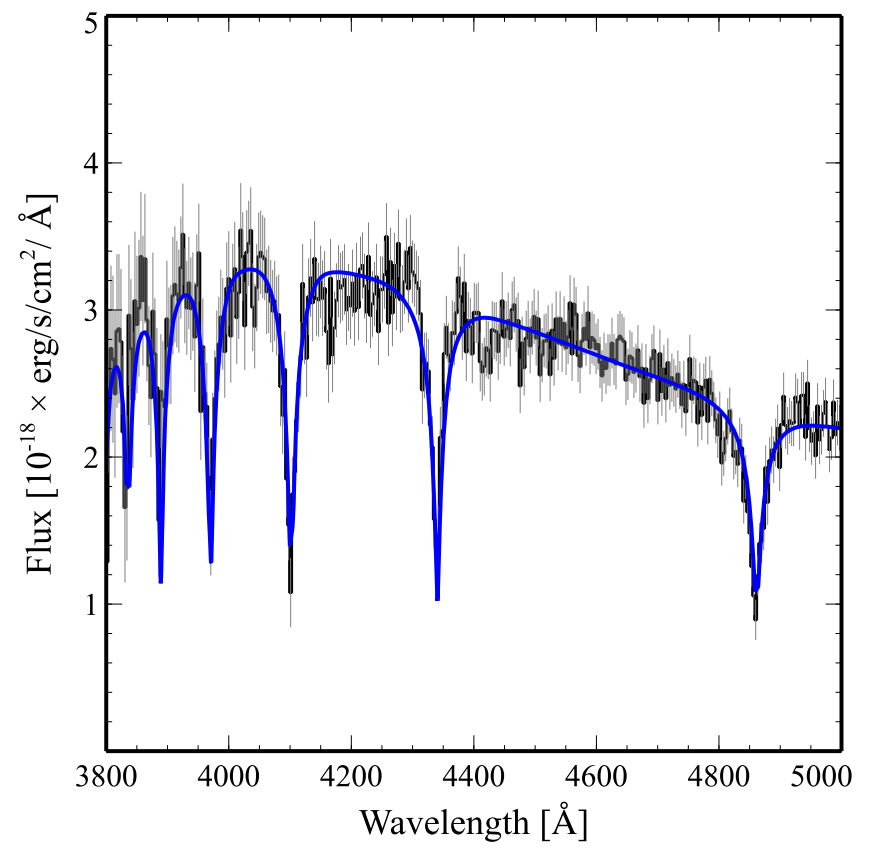

Figure 2. Zero-velocity median combination of the spectra shown in Figure 1, (binned by 4 for clarity). The solid blue line shows the best-fit spectrum. Measurement uncertainties are shown in gray.

of DA model atmospheres (Koester 2008) to a single spectrum, used the best-fit model as a template to shift and average the spectra, and then refined the template by fitting the average spectrum.

Figure 1 shows the radial velocities of the WD relative to that of the reference star, as a function of orbital phase. Unfortunately, the uncertainties of individual measurements $\left(>23 \mathrm{~km} \mathrm{~s}^{-1}\right)$ are comparable to the expected amplitude of the orbital velocity of $K_{\mathrm{c}}=1.39 / 0.2752 \pi x / P_{\mathrm{b}}=47.99 \mathrm{~km} \mathrm{~s}^{-1}$, thereby not allowing for a precise independent determination of the mass ratio. After excluding eight measurements with uncertainties larger than $60 \mathrm{~km} \mathrm{~s}^{-1}$ and an extreme outlier with $\delta v=442 \pm 22 \mathrm{~km} \mathrm{~s}^{-1}$, a fit to the orbit using the timing ephemeris of the pulsar-with $T_{0}=56794.0931864 \mathrm{MJD}, P_{\mathrm{b}}=$ 32.00140 day, $e=0.129274$, and $\omega=277.16764$ degreesyields an orbital velocity semi-amplitude of $K_{\mathrm{c}}=81 \pm$ $23 \mathrm{~km} \mathrm{~s}^{-1}$ and a systemic velocity of $\Delta \gamma=+137.2 \mathrm{~km} \mathrm{~s}^{-1}$ relative to the reference star, with $\chi_{\text {red }}=1.9$ for 15 degrees of freedom. Combined with the orbital parameters of the pulsar constrained with timing, the inferred mass ratio is $q \equiv$ $M_{\mathrm{PSR}} / M_{\mathrm{c}}=8.5 \pm 2.4$.

The radial velocity of the reference star varied as much as $44 \mathrm{~km} \mathrm{~s}^{-1}$ among the 17 observations, which is considerably larger than the formal uncertainties. However, for the latter, we find no evidence for binarity. A visual inspection of the through-slit pointing frames taken before the science exposures suggests that the most likely cause is differential diffraction due to minor displacements of the star inside the slit. The inferred mean radial velocity is $\gamma_{\text {ref }}=-93 \pm 14 \mathrm{~km} \mathrm{~s}^{-1}$, which differs by $25 \mathrm{~km} \mathrm{~s}^{-1}$ compared to the velocity found from the wide-slit spectra, $\gamma_{\text {ref, wide }}=-117 \pm 4 \mathrm{~km} \mathrm{~s}^{-1}$. Conservatively adopting $\gamma_{\text {ref }}=-117 \pm 25 \mathrm{~km} \mathrm{~s}^{-1}$ yields an absolute systemic velocity of $\gamma=20 \pm 34 \mathrm{~km} \mathrm{~s}^{-1}$ for PSR J2234+0611, relative to the solar system barycenter.

\subsection{Atmospheric Parameters}

The zero-velocity average of the 17 best spectra mentioned above is shown in Figure 2. The spectrum is that of a typical pure-hydrogen WD, thereby confirming our initial photometric classification.

We fitted the spectrum using a grid of DA model atmospheres covering the range 6000-10000 K with a step size of $250 \mathrm{~K}$ in $T_{\text {eff }}$, and $6.0-8.0 \mathrm{dex}$ with a step size of $0.25 \mathrm{dex}$ in $\log g$ (Koester 2008). For the fit, we convolved each model using a Gaussian kernel with a dispersion equal to that of the average seeing truncated at the slit width. We also allowed for the normalization to vary with wavelength using a third-degree polynomial. The fit yields an effective temperature of $T_{\text {eff }}=8749 \pm 120 \mathrm{~K}$ and a surface gravity of $\log g=7.25 \pm 0.15 \mathrm{dex}$. To get an estimate for the systematics we varied the degree of the normalization polynomial, the parameters of the convolution kernel and the spectral range used for the fit. These fits were overall consistent with each other, with a scatter slightly larger than the $1 \sigma$ formal uncertainties. To compensate for that we adopt $T_{\text {eff }}=8750 \pm 200 \mathrm{~K}$ and $\log g=7.25 \pm 0.20$ dex for all our calculations below.

The derived atmospheric parameters place the WD in a regime where convective atmospheric effects are known to produce systematic errors in one-dimensional (1D) model atmospheres (Tremblay et al. 2011). Using the numerical estimates of Tremblay et al. (2013, 2015), we find that the "true" atmospheric parameters are $T_{\text {eff }}=8600 \pm 190 \mathrm{~K}$ and $\log g=6.97 \pm 0.22$ dex.

\subsection{WD Radius and Model-independent Surface Gravity}

Using the temperature estimate, the observed flux, and the parallactic distance, we can obtain an estimate of the WD radius via

$$
\begin{aligned}
& m_{\lambda}-5 \log _{10}(d / 10 \mathrm{pc})-A_{\lambda}= \\
& \quad-5 \log \left(R_{\odot} / 10 \mathrm{pc}\right)-5 \log _{10}\left(R / R_{\odot}\right)-2.5 \log _{10} F_{\lambda}+c_{\lambda},
\end{aligned}
$$

where $m_{\lambda}$ is the apparent magnitude in band $\lambda, A_{\lambda}$ is the corresponding reddening, $F_{\lambda}$ is the emitted luminosity per unit area integrated over the bandpass and $c_{\lambda}$ is the zero-point of the filter. Convolving the best-fit atmospheric model with the SDSS- $g$ bandpass (Gunn et al. 1998) $)^{9}$ yields $F_{\mathrm{g}}=4.9745 \times 10^{7} \mathrm{erg} \mathrm{cm}^{-2} \mathrm{~s}^{-1}$. For the reddening, the galactic-extinction map of Schlegel et al. (1998) gives $A_{\mathrm{g}}=0.481$ for the total extinction along the line of sight, which however can be considerably smaller given the proximity of the system. If we conservatively adopt $A_{\mathrm{g}}=0.16-0.481$ we find $R_{\mathrm{WD}}=0.024_{-0.002}^{+0.004} R_{\odot}$, where the uncertainty also takes into account the parallactic and photometric errors.

Together with the WD mass derived in Paper II, the former estimate yields $\log _{10} g=7.11_{-0.16}^{+0.08}$ dex for the surface gravity, consistent with the previous spectroscopic estimate (Section 2.2).

\footnotetext{
9 http://svo2.cab.inta-csic.es/svo/theory/fps3/index.php?id=SLOAN/ SDSS.g
} 

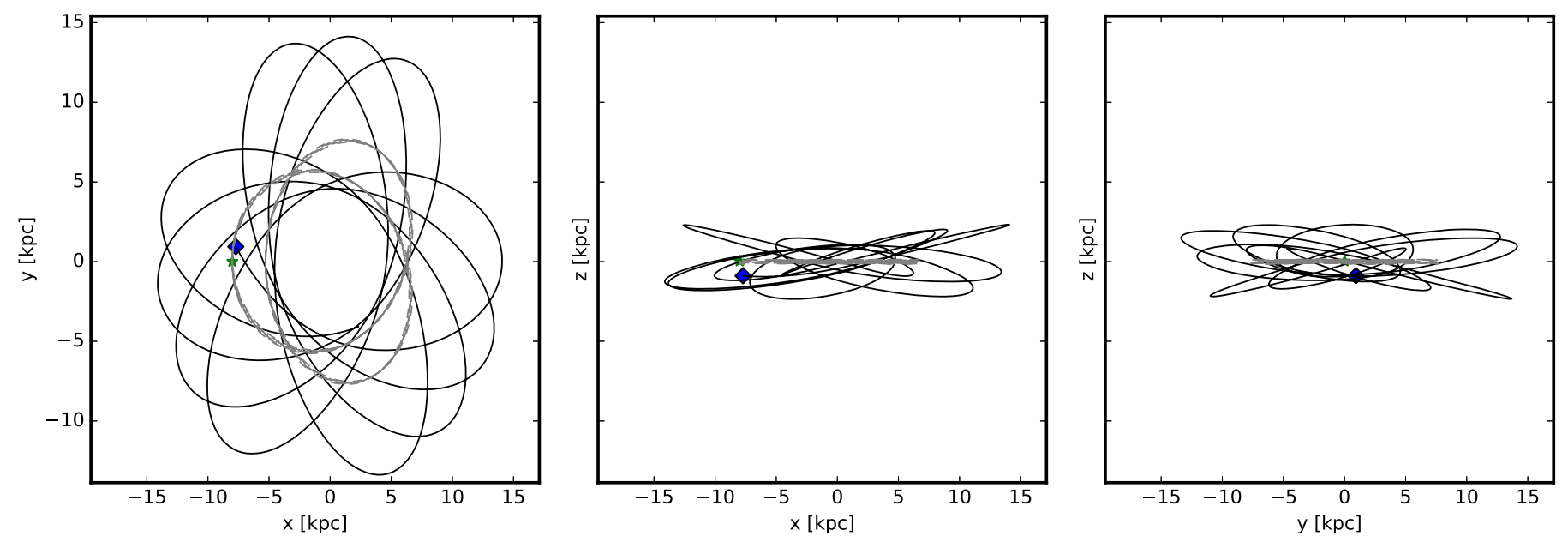

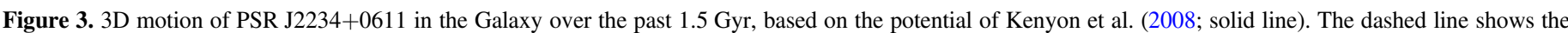
orbit of the Sun. The current positions of PSR J2234+0611 and the Sun are marked with a diamond and a star, respectively. All axes are in kiloparsecs.

\subsection{Kinematics}

The three-dimensional (3D) velocity information from the combined timing and spectroscopic analysis allows us to calculate the orbit of the system inside the gravitational potential of the Galaxy. Figure 3 shows the system's orbit based on the Kenyon et al. (2008) empirical model for the Galactic potential, over the past 1.5 Gyr. We find that the orbit of the system is highly eccentric with a galactocentric distance varying from 4 to $15 \mathrm{kpc}$ and a vertical component extending to $\sim 2 \mathrm{kpc}$. The velocity relative to the local standard of rest when the system crosses the galactic plane $(Z=0)$ ranges from $\sim 55$ to $130 \mathrm{~km} \mathrm{~s}^{-1}$. In Section 4 we discuss the implications of these constraints for the formation of the system.

\subsection{Spectrophotometry}

Finally, we computed synthetic magnitudes by convolving our spectra with the SDSS $g$-filter response. Uncertainties were estimated using a Monte-Carlo approach, where multiple realizations of the spectra were convolved and compared with each other. The differential magnitude relative to the reference star is shown in Figure 4. The observed peak-to-peak difference of $\sim 0.5 \mathrm{mag}$ is more than an order of magnitude larger than the formal uncertainties. We find no compelling evidence for correlation with the orbital motion. Given the differential refraction effects that likely polluted our radial velocities, it is possible that part of the scatter can be accounted for by slit displacements. However, we find no definite correlation with this effect. Another possible cause is an intrinsic luminosity change of the LMWD, which could be due to pulsational instabilities, like those seen in ZZ-Ceti stars and extremely lowmass WDs (e.g., Kilic et al. 2015, and references therein).

\section{DISCUSSION}

The observed and derived physical parameters of the system are summarized in Table 2. PSR J2234+0611 is the first known case of a Galactic eMSP with an LMWD companion. The combination of precision timing measurements (Paper II) and phase-resolved spectroscopy make the binary a unique test bed for stellar evolution physics. In the remainder of this section we explore the ramifications of our work for LMWD models and place constraints on the evolution of the system.
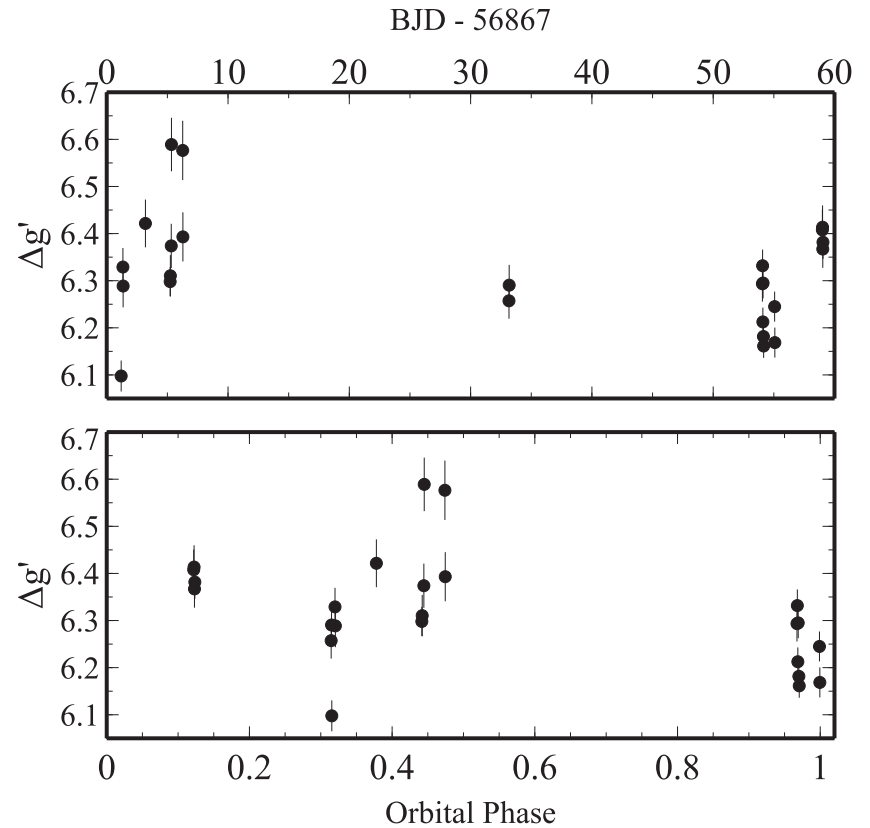

Figure 4. g-band differential synthetic magnitudes for the companion to PSR J2234+0611 displayed as a function of time (upper) and orbital phase (lower).

\subsection{Origin and Evolution}

One question we can address directly is or not whether PSR J2234+0611 evolved from an interacting binary. First, the WD nature of the companion is consistent with the expectations for LMXB evolution. For systems within this orbitalperiod range, the mass transfer starts while the donor star ascends the red-giant branch. For solar-metallicity progenitors, the theoretical mass-orbital period relation of Tauris \& Savonije (1999) predicts a mass of $0.29 M_{\odot}$ for the WD, which is slightly larger than the observed value. However, the marginal difference could be attributed to other factors such as initial ZAMS composition and/or remaining uncertainties in the evolution models of LMXBs.

A second diagnostic is the system's motion in the Galaxy. PSR J2234+0611 has a peculiar velocity of $\sim 31 \mathrm{~km} \mathrm{~s}^{-1}$, relative to the local standard of rest, which is typical for 
Table 1

Comparison of Theories for the Formation of Eccentric MSPs

\begin{tabular}{|c|c|c|c|c|c|c|}
\hline Theory & Orbital Periods & Companion & Companion Mass & Pulsar Mass & Notes & References \\
\hline Triple System & unbound & MS or WD & unbound & unbound & & \\
\hline Delayed AIC & 10-60 days & LMWD & $0.25-0.35 M_{\odot}$ & $1.35 M_{\odot}$ & The implosion is symmetric, leading to small systemic velocities & Freire \& Tauris (2014) \\
\hline Quark Phase Transition & unbound & LMWD & unbound & $\sim 1.8 M_{\odot}$ & & Jiang et al. (2015) \\
\hline CB Disk & 15-30 days & LMWD & $0.22-0.35 M_{\odot}$ & unbound & Small number of circular binaries within this orbital period range & Antoniadis (2014) \\
\hline
\end{tabular}


LMXB descendants (Lorimer 2005). Furthermore, the "crossing velocity" (at $Z=0$ ) ranges from 34 to $130 \mathrm{~km} \mathrm{~s}^{-1}$, which is in broad agreement with the predictions for core-collapse supernovae (e.g., Scheck et al. 2006 and references therein).

Therefore, even though a triple-origin scenario cannot be ruled out conclusively, the consistency of the properties of the PSR J2234+0611 system with Galactic-field MSPs strongly support the binary evolution hypothesis.

Regarding the specific mechanism that gave rise to the high eccentricity, further evidence comes from the pulsar and WD masses. As we briefly discussed in Section 1, one possibility is that the MSP formed via a spontaneous phase transition. Assuming a symmetric implosion with a negligible momentum kick, the observed masses and eccentricity can be linked directly to the amount of mass radiated during the explosion, $\Delta M=e\left(M_{\mathrm{PSR}}+M_{\mathrm{c}}\right)=0.215 M_{\odot}$, and the progenitor mass, $M_{\mathrm{PSR}}+\Delta M=1.60 M_{\odot}$. These constraints disfavor the scenario proposed by Jiang et al. (2015), in which an NS transforms to a quark star, as we would expect such a transition to occur at high energy densities, relevant for NS masses $>1.8 M_{\odot}($ Jiang et al. 2015, c.f. Table 1).

An alternative mechanism proposed by Freire \& Tauris (2014) theorizes that eMSPs form indirectly from a rotationally delayed accretion-induced collapse (AIC) of a massive WD. First, because WDs require a fine-tuned mass transfer rate to grow in mass (Nomoto \& Kondo 1991; Chen et al. 2011; Tauris et al. 2013), the resultant systems are expected to have orbital periods in the 10-60 day range, in agreement with the known eMSPs. Freire \& Tauris (2014) propose that the AIC could in principle be delayed until after the cessation of mass transfer, due to the rapid rotation of the WD progenitor. The radiated gravitational binding energy during the AIC would therefore induce the observed high eccentricities as no further circularization is expected from the two compact objects.

To first order, the imploding WD should only be slightly above the Chandrasekhar limit (Nomoto \& Kondo 1991; Dessart et al. 2006), and therefore collapse into a pulsar with a gravitational mass in the $1.22-1.31 M_{\odot}$ range, assuming small amounts of baryonic mass loss during the transition (Freire \& Tauris 2014). The constraint on the progenitor mass, and the peculiar velocity of $\sim 31 \mathrm{~km} \mathrm{~s}^{-1}$ inferred here, therefore excludes this simple version of the RD-AIC mechanism. However, it is possible that if one relaxes the assumption for rigid rotation for the progenitor WD (Yoon \& Langer 2004), the the mass of the resulting pulsar could be higher. In addition, strong magnetic fields may also play an important role (see discussion in Freire \& Tauris 2014). In such a case, an asymmetric mass loss would still be required to explain both the large systemic velocity and eccentricity (Freire \& Tauris 2014).

For the AIC to be successful, the loss of angular momentum should happen on a timescale larger than the Debye cooling time, to allow for efficient ${ }^{24} \mathrm{Mg}$ and ${ }^{20} \mathrm{Ne}$ electron captures instead of oxygen deflagration of the (hot) core (Tauris et al. 2013; Freire \& Tauris 2014). Since super-Chandrasekhar WDs crystalize within $\sim 10^{8}$ year (e.g., Bergeron et al. 1995), the inferred LMWD cooling age of $\sim 1.5 \mathrm{Gyr}$ (see below) is therefore also consistent with this scenario. In summary, albeit fine-tuned, an AIC mechanism cannot be ruled out completely. Mass measurements for other eMSPs are necessary to further constrain this scenario.

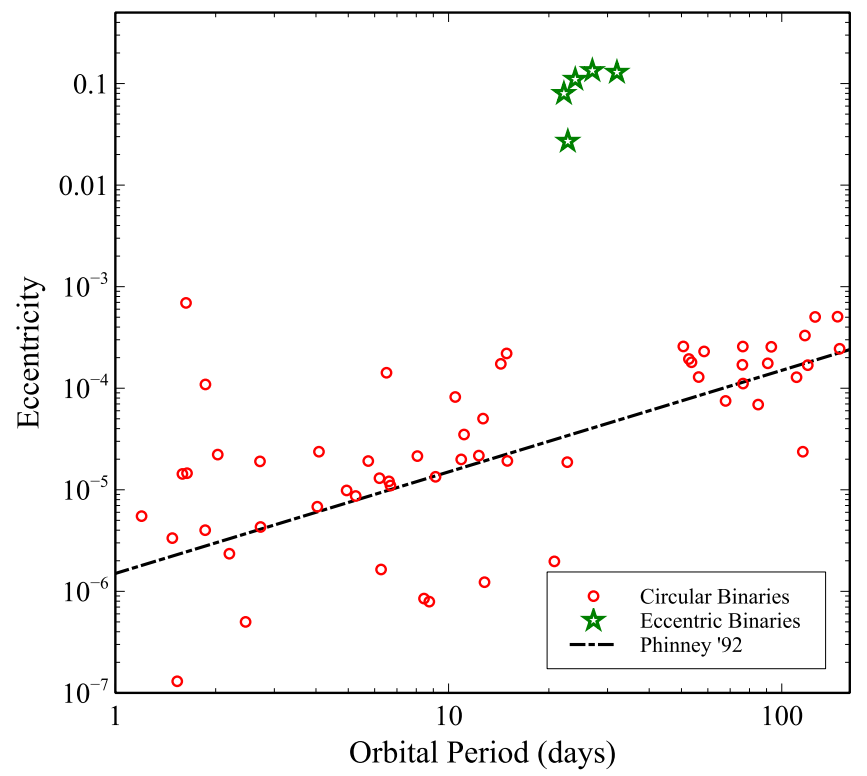

Figure 5. Orbital periods and eccentricities of binary MSPs with orbital periods between 1 and 150 days. Circular binaries (red circles) are taken from the ATNF pulsar catalog (Manchester et al. 2005). The eMSPs shown are (from left to right) PSRs J1618-3919 (Bailes 2010), J0955-6150 (Camilo et al. 2015), J1950+2414 (Knispel et al. 2015), J1946+3417 (Barr et al. 2013), and J2234+0611 (Deneva et al. 2013, this work). The theoretical prediction of Phinney (1992) is also shown as a dashed line. Note that the apparent increased scatter at small orbital periods may be artificially induced by covariances between the eccentricity and Shapiro delay in the timing model.

Finally, we consider the possibility of eccentricity pumping via a short-term interaction between the post-LMXB system and a circumbinary (CB) disk (Antoniadis 2014). Such a disk can be fueled by material escaping the proto-WD due unstable $\mathrm{CNO}$ burning (H-flash). Because $\mathrm{H}$-flashes are expected only for a limited range of WD masses $\left(\sim 0.2-0.35 M_{\odot}\right.$, e.g., Althaus et al. 2013; Antoniadis 2013; Istrate et al. 2014), this mechanism predicts a statistical correlation between the eccentricity and orbital period that is applicable to all MSP systems. This indeed seems to be the case, as all eMSPs, including PSR J2234+0611, have orbital periods between 22 and 32 days (c.f. Figure 5 and references in the caption) - a regime where circular MSPs have yet to be discovered. In the analytic framework considered by Antoniadis (2014), the observed eccentricities are linked to the CB-disk mass and lifetime, as well as the initial eccentricity (Artymowicz et al. 1991; Dermine et al. 2013). This work finds that an eccentricity of $e \sim 0.13$ for the observed orbital separation requires $M_{\mathrm{d}} \tau_{\mathrm{d}} \simeq 75 M_{\odot}$ yr for the disk mass and lifetime. For the typical mass loss of $10^{-4} M_{\odot}$ occurring during an H-flash (Antoniadis 2014), this yields $\tau_{\mathrm{d}} \simeq 50,000$ year, which is much shorter than the inferred cooling age. In addition, it is possible that the interaction is even more efficient, if the dependence on the eccentricity of the disk's central cavity is weak, as found in recent high-resolution shock-capturing simulations (D'Orazio et al. 2013).

The CB-disk mechanism poses no additional constraint on the eMSP masses, apart from those expected from nuclear, core-collapse, and accretion physics (Sukhbold et al. 2015).

The observed pulsar mass of $1.39 M_{\odot}$ implies a small accretion efficiency for the recycling process. Following Antoniadis et al. (2012), if we indeed interpret the system as a direct descendant of a sub-Eddington mass-transferring 
binary, the donor's initial mass must have been $\gtrsim 1 M_{\odot}$, in order for the star to reach the red-giant branch within a Hubble time. Assuming a pulsar birth mass $\geqslant 1.17 M_{\odot}$ (Martinez et al. 2015), the former considerations therefore imply an efficiency of $\alpha<30 \%$. A more "typical" NS birth mass of $\sim 1.35 M_{\odot}$ (Özel et al. 2012), however, yields $\alpha<6 \%$.

\subsection{Prospects for LMWD Physics}

PSR J2234+0611 is only the third pulsar-LMWD binary with model-independent mass and radius constraints (see Antoniadis et al. 2012; Antoniadis 2013; Kaplan et al. 2014, for earlier discussions), making the system a valuable test bed for WD atmospheric and cooling properties. Unfortunately, the precision of our spectroscopic measurements does not suffice for a detailed confrontation with theoretical model atmospheres. For instance, both the 1D- and 3D-corrected atmospheric properties are consistent with the model-independent estimates within formal uncertainties (see Sections 3.2 and 3.3). Further spectroscopic observations, which will increase the signal-to-noise of the spectrum, are therefore required to draw any further conclusions.

The timing mass and radius constraints are consistent with cooling models for WDs with relatively thin hydrogen atmospheres. For instance, the recent models of Althaus et al. (2013) yield a radius of $R=0.022 R_{\odot}$ for a $0.275 M_{\odot}$ WD, which is in perfect agreement with our estimate derived in Section 3.3. The same models yield a cooling age of $\tau_{\mathrm{c}}=1.5 \mathrm{Gyr}$. The former should also be comparable to the age of the system as the expected evolution timescale of a $0.275 M_{\odot}$ proto-WD before it settles on the cooling branch is small (Istrate et al. 2014).

Perhaps more important is the fact that PSR J2234+0611 lies close to the low-mass extension of the ZZ-Ceti instability strip, as derived empirically by Gianninas et al. (2015). The variability of $\sim 0.5 \mathrm{mag}$ seen in our data set is large compared to what is found for other pulsating LMWDs, like for instance PSR J1738+0333 (Kilic et al. 2015), and more similar to classical ZZ-Ceti stars. If PSR J2234+0611 indeed pulsates due to excitation of non-radial $g$-modes (Córsico et al. 2012; Van Grootel et al. 2013), we would expect a longer dominant periodicity due to the lower temperature compared to PSR J1738+0333 (Antoniadis et al. 2012). Further highcadence photometric observations have the potential to probe the WD interior in detail and help infer (and calibrate) the atmospheric composition, hydrogen mass, and interior convective properties. Even if pulsations are not confirmed, the system will place strong constraints on the exact location of the instability strip in a poorly constrained regime.

\section{CONCLUSIONS}

We have presented phase-resolved spectroscopic observations of the companion to PSR J2234+0611. Our data unabiguously identify the star as a low-mass He WD-the first found orbiting a galactic-field MSP with non-zero eccentricity.

We find that the WD mass is consistent with the expectations for LMXB evolution and strongly disfavors a triple-star formation hypothesis. In addition, the pulsar mass (Table 2) contradicts the quark-nova formation theory proposed by Jiang
Table 2

Properties of the PSR J2234+0611 System

\begin{tabular}{|c|c|}
\hline Observed Parameter & Value \\
\hline Reference epoch (MJD) & 56794 \\
\hline Time of ascending node (MJD) & $56794.09318642(9)$ \\
\hline$\mu_{\alpha}\left(\operatorname{mas~yr}^{-1}\right)$ & $+25.3896(271)$ \\
\hline$\mu_{\delta}\left(\operatorname{mas~} \mathrm{yr}^{-1}\right)$ & $+9.4816(619)$ \\
\hline Parallax, $\pi$ (mas) & $0.7423(279)$ \\
\hline Orbital period, $P_{b}$ (days) & $32.001401609(14)$ \\
\hline Eccentricity, $e$ & 0.1292740499 \\
\hline Inferred Parameter & Value \\
\hline Pulsar mass, $M_{\mathrm{PSR}}\left(M_{\odot}\right)$ & $1.39(1)$ \\
\hline WD mass, $M_{\mathrm{c}}\left(M_{\odot}\right.$, spectroscopy $)$ & $0.275(8)$ \\
\hline Temperature (K; 3D-corrected) & $8600(190)$ \\
\hline Surface gravity (log $g, 3 \mathrm{D}$-corrected) & $6.97(22)$ \\
\hline Surface gravity (log $g, \pi+$ photometry) & $7.11_{-0.16}^{+0.08}$ \\
\hline Photometry, $g$-band & $22.17(10)$ \\
\hline Semi-amplitude of radial velocity, $K_{\mathrm{WD}}\left(\mathrm{km} \mathrm{s}^{-1}\right)$ & $81(23)$ \\
\hline Systemic radial velocity, $\gamma\left(\mathrm{km} \mathrm{s}^{-1}\right)$ & $-20(34)$ \\
\hline Transverse velocity, $v_{T}\left(\mathrm{~km} \mathrm{~s}^{-1}\right)$ & 179 \\
\hline 3D velocity amplitude $\left(\mathrm{km} \mathrm{s}^{-1}\right)$ & 180 \\
\hline Mass ratio, $q$ (timing) & 5.05 \\
\hline WD radius (photometry) $\left(R_{\odot}\right)$ & $0.024_{-0.002}^{+0.004}$ \\
\hline Cooling age, $\tau_{\mathrm{c}}(\mathrm{Gyr})$ & 1.5 \\
\hline
\end{tabular}

et al. (2015). Combined with the inferred peculiar velocity, it also poses stringent constraints on the rotationally delayed AIC hypothesis of Freire \& Tauris (2014), as the latter requires both a differentially rotating super-Chandrasekhar-mass WD progenitor and asymmetric mass loss at birth. On the other hand, we find the mechanism of Antoniadis (2014)—which proposes eccentricity pumping via interaction with a transient $\mathrm{CB}$ diskto be consistent with all observed and inferred parameters. If PSR J2234+0611 indeed originates from an LMXB, the low pulsar mass implies a small accretion efficiency during recycling of at most $30 \%$, with a more likely value close to $6 \%$.

Finally, we find tentative evidence for pulsations, which, together with the independent constraints on the stellar radius and mass, transform the system into a unique test bed for LMWD evolution. We are looking forward to further detailed spectroscopy and high-cadence photometry that will allow for a detailed and unprecedented confrontation with models.

This work is based on observations made with ESO Telescopes at the Paranal Observatories under programme ID 093.D-0108(A). We wish to thank the ESO astronomers-onduty for the excellent execution of the observations.

J. A. is a Dunlap Fellow at the Dunlap Institute for Astronomy and Astrophysics at the University of Toronto. The Dunlap Institute is funded by an endowment established by the David Dunlap family and the University of Toronto. P. C. C. F. P. F. acknowledges financial support by the European Research Council for the ERC Starting grant AST 1108753. J. S. D. was supported by the NASA's Fermi Guest Investigator program.

We have made extensive use of NASA's Astrophysics Data System and Astropy, a community-developed core Python package for Astronomy. 


\section{REFERENCES}

Althaus, L. G., Miller Bertolami, M. M., \& Córsico, A. H. 2013, A\&A, 557, A19

Antoniadis, J. 2013, PhD thesis, Univ. Bonn

Antoniadis, J. 2014, ApJL, 797, L24

Antoniadis, J., Freire, P. C. C., Wex, N., et al. 2013, Sci, 340, 448

Antoniadis, J., van Kerkwijk, M. H., Koester, D., et al. 2012, MNRAS, 423, 3316

Appenzeller, I., Fricke, K., Furtig, W., et al. 1998, Msngr, 94, 1

Archibald, A. M., Stairs, I. H., Ransom, S. M., et al. 2009, Sci, 324, 1411

Artymowicz, P., Clarke, C. J., Lubow, S. H., \& Pringle, J. E. 1991, ApJL, 370, L35

Astropy Collaboration, Robitaille, T. P., Tollerud, E. J., et al. 2013, A\&A, 558, A33

Bailes, M. 2010, NewAR, 54, 80

Barr, E. D., Champion, D. J., Kramer, M., et al. 2013, MNRAS, 435, 2234

Bejger, M., Fortin, M., Haensel, P., \& Zdunik, J. L. 2011, A\&A, 536, A87

Bergeron, P., Saumon, D., \& Wesemael, F. 1995, ApJ, 443, 764

Bhattacharya, D., \& van den Heuvel, E. P. J. 1991, PhRvD, 203, 1

Camilo, F., Kerr, M., Ray, P. S., et al. 2015, ApJ, 810, 85

Champion, D. J., Ransom, S. M., Lazarus, P., et al. 2008, Sci, 320, 1309

Chen, W.-C., Liu, X.-W., Xu, R.-X., \& Li, X.-D. 2011, MNRAS, 410, 1441

Córsico, A. H., Romero, A. D., Althaus, L. G., \& Hermes, J. J. 2012, A\&A, 547, A96

Deneva, J. S., Stovall, K., McLaughlin, M. A., et al. 2013, ApJ, 775, 51

Dermine, T., Izzard, R. G., Jorissen, A., \& Van Winckel, H. 2013, A\&A, 551, A50

Dessart, L., Burrows, A., Ott, C. D., et al. 2006, ApJ, 644, 1063

D’Orazio, D. J., Haiman, Z., \& MacFadyen, A. 2013, MNRAS, 436, 2997

Eisenstein, D. J., Weinberg, D. H., Agol, E., et al. 2011, AJ, 142, 72

Freire, P. C. C., Bassa, C. G., Wex, N., et al. 2011, MNRAS, 412, 2763

Freire, P. C. C., \& Tauris, T. M. 2014, MNRAS, 438, L86

Gianninas, A., Kilic, M., Brown, W. R., Canton, P., \& Kenyon, S. J. 2015, ApJ, 812, 167

Gunn, J. E., Carr, M., Rockosi, C., et al. 1998, AJ, 116, 3040

Horne, K. 1986, PASP, 98, 609

Husser, T.-O., Wende-von Berg, S., Dreizler, S., et al. 2013, A\&A, 553, A6

Istrate, A. G., Tauris, T. M., Langer, N., \& Antoniadis, J. 2014, A\&A, 571, L3
Jiang, L., Li, X.-D., Dey, J., \& Dey, M. 2015, ApJ, 807, 41

Kaplan, D. L., van Kerkwijk, M. H., Koester, D., et al. 2014, ApJL, 783, L23

Kenyon, S. J., Bromley, B. C., Geller, M. J., \& Brown, W. R. 2008, ApJ, 680,312

Kilic, M., Hermes, J. J., Gianninas, A., \& Brown, W. R. 2015, MNRAS, 446, L26

Knispel, B., Lyne, A. G., Stappers, B. W., et al. 2015, ApJ, 806, 140

Koester, D. 2008, arXiv:0812.0482

Lorimer, D. R. 2005, LRR, http://relativity.livingreviews.org/Articles/lrr2005-7/

Manchester, R. N., Hobbs, G. B., Teoh, A., \& Hobbs, M. 2005, AJ, 129, 1993

Martinez, J. G., Stovall, K., Freire, P. C. C., et al. 2015, ApJ, 812, 143

Nomoto, K., \& Kondo, Y. 1991, ApJL, 367, L19

Özel, F., Psaltis, D., Narayan, R., \& Santos Villarreal, A. 2012, ApJ, 757, 55

Perets, H. B., \& Kratter, K. M. 2012, ApJ, 760, 99

Phinney, E. S. 1992, Phil. Trans.:Phys. Sc.\& Eng., 341, 39

Pijloo, J. T., Caputo, D. P., \& Portegies Zwart, S. F. 2012, MNRAS, 424, 2914

Portegies Zwart, S., van den Heuvel, E. P. J., van Leeuwen, J., \& Nelemans, G. 2011, ApJ, 734, 55

Scheck, L., Kifonidis, K., Janka, H.-T., \& Müller, E. 2006, A\&A, 457, 963

Schlegel, D. J., Finkbeiner, D. P., \& Davis, M. 1998, ApJ, 500, 525

Serenelli, A. M., Althaus, L. G., Rohrmann, R. D., \& Benvenuto, O. G. 2001, MNRAS, 325, 607

Sukhbold, T., Ertl, T., Woosley, S. E., Brown, J. M., \& Janka, H.-T. 2015, arXiv: 1510.04643

Taam, R. E., \& van den Heuvel, E. P. J. 1986, ApJ, 305, 235

Tauris, T. M. 2011, in ASP Conf. Ser. 447, Evolution of Compact Binaries, ed. L. Schmidtobreick, M. R. Schreiber, \& C. Tappert (San Francisco, CA: ASP), 285

Tauris, T. M., Sanyal, D., Yoon, S.-C., \& Langer, N. 2013, A\&A, 558, A39

Tauris, T. M., \& Savonije, G. J. 1999, A\&A, 350, 928

Tremblay, P., Ludwig, H., Steffen, M., Bergeron, P., \& Freytag, B. 2011, A\&A, 531, L19

Tremblay, P.-E., Gianninas, A., Kilic, M., et al. 2015, ApJ, 809, 148

Tremblay, P.-E., Ludwig, H.-G., Steffen, M., \& Freytag, B. 2013, A\&A, 559, A104

Van Grootel, V., Fontaine, G., Brassard, P., \& Dupret, M.-A 2013, ApJ, 762,57

Yoon, S.-C., \& Langer, N. 2004, A\&A, 419, 623 